\title{
Experiments and Theory for Two Grids Turbulence
}

\section{Harry E. Schulz \\ heschulz@sc.usp.br \\ Johannes G. Janzen \\ jgersonj@gmx.net}

Karine Cristiane de 0. Souza

Escola de Engenharia de São Carlos Universidade de São Paulo - USP 13566-590 São Carlos, SP, Brazil
Turbulence generated by a pair of oscillating grids in a water tank is investigated using the digital particle image velocimetry technique. Vertical and horizontal components of fluctuating velocities are measured. Experimental profiles of the turbulent kinetic energy $k$, and the energy dissipation rate, $\xi$, obtained from the fluctuating velocities, are presented and compared with theoretical predictions. Other properties of interest, such as the length scale and the turbulent viscosity, obtained in the present study, are also reported. The turbulence generated with the pair of grids may be classified as "nearly isotropic" and theoretically the flow may be analyzed using the $k$ - $\varepsilon$ model. The experimental data agree well with the theoretical predictions. Also data of different sources found in literature, for pairs of vibrating grids, are compared with the present experimental results and theoretical predictions, showing good agreement. The studies conducted at the Laboratory of Environmental Hydraulics from the Department of Hydraulics and Sanitary Engineering, EESC/USP, permits to affirm that "double oscillating grids" turbulence can be viewed as nearly isotropic.

Keywords: Grid-turbulence, oscillating grids, turbulence models, $k$-Emodel

\section{Introduction}

The study of isotropic turbulence began with Taylor, who defined it as the condition in which all statistical properties are independent of direction. Since then, considered the "simplest kind" of turbulence (in the sense that the least number of parameters is necessary to describe it), isotropic turbulence has been broadly studied aiming a better understanding of turbulence itself and its relation with correlated transport phenomena. It has been used in theoretical treatments of turbulence with more complex flows (Townsend, 1976). For many decades, "experimental isotropic turbulence" has been "generated" in different ways. However, despite all the effort, this type of turbulence can only be approximated, as the perfect isotropy is only a theoretical hypothesis and impossible to be reached experimentally (Srdic et al. 1996). Therefore, "experimental isotropy" is called "nearly-isotropic turbulence". Some authors used grid-turbulence to attain a steady condition of nearly-isotropic turbulence. Among them are De Silva and Fernando (1994), Shy et al (1996), Brunk et al. (1996), Thompson and Turner (1975), Hopfinger and Toly (1976), Souza (2002), Janzen (2003) and Janzen and Schulz (2003). In the oscillating grid configuration, a grid (usually square) of mesh size $M$ oscillates around its mean position, with amplitude $S$ (the stroke) at a frequency $f$ in a tank. Because the turbulence intensities decay rapidly as the distance from the grid increases, Villermaux et al. (1995), Srdic et al. (1996) and Shy et al. (1996), in independent studies, proposed a two-grid configuration, already being object of study by Shy et al. (1997), Ott and Mann (2000), and Janzen (2003). In this study, the digital particle image velocimetry (DPIV) technique is employed to investigate the turbulence produced by a pair of oscillating grids. The fields for turbulent kinetic energy and its dissipation rate have been compared with predictions of analytical solutions of the $k-\varepsilon$ turbulence model, proposed by Schulz and Chaudhry (1998, 1999), and Schulz (2001). Good agreement between theory and experimental data is observed. Other characteristic quantities such as length scale, Reynolds shear stress and eddy viscosity are also presented.

Presented at ETT $2004-4^{\text {th }}$ Spring School on Transition and Turbulence September $27^{\text {th }}$ - October $1^{\text {st }}, 2004$, Porto Alegre. RS. Brazil.

Paper accepted: May, 2005. Technical Editor: Aristeu da Silveira Neto.

\section{Nomenclature}

$C_{D}=$ coefficient for the evaluation of $\varepsilon$, dimensionless.

$C_{2 \varepsilon}=k-\varepsilon$ model constant for the $\varepsilon$-equation., dimensionless.

$C_{\mu}=k-\varepsilon$ model constant for $v_{t}$, dimensionless.

$f=$ oscillation frequency, $\mathrm{Hz}$.

$f(r)=$ longitudinal correlation function, dimensionless.

$F=$ Elliptic integral.

$F_{m}=$ Matsunaga et al. transformed space dimension, $s / m$.

$g(r)=$ transversal correlation function, dimensionless.

Hyp $2 F 1=$ Hypergeometric function.

$j=$ coefficient of the $\varepsilon$ equation, dimensionless.

$k=$ turbulent kinetic energy per unit mass, $\mathrm{m}^{2} / \mathrm{s}^{2}$.

$\bar{k}=$ modified turbulent kinetic energy per unit mass, $\mathrm{m}^{2} / \mathrm{s}^{2}$.

$k^{*}=$ nondimensional turbulent kinetic energy.

$L=$ half distance between oscillating grids, $m$.

$L_{t}=$ Turbulence length scale, $m$.

$L_{f}=$ Turbulence length scale, $m$.

$L_{g}=$ Turbulence length scale, $m$.

$m=$ parameter of the elliptic integral, dimensionless.

$M=$ mesh size.

$N=$ number of images, dimensionless.

$r=$ distance between two points in the fluid, $m$.

$R e=$ Reynolds number , dimensionless.

$s=$ dimensionless distance.

$S=$ Stroke, $m$.

$u=R M S$ value of the $x$-component of the velocity (turbulence intensity, defined according to Brodkey, 1967), $\mathrm{m} / \mathrm{s}$.

$u^{\prime}=$ fluctuation of the $x$-component of the velocity, $\mathrm{m} / \mathrm{s}$.

$w=R M S$ value of the $z$-component of the velocity (turbulence intensity, defined according to Brodkey, 1967), $\mathrm{m} / \mathrm{s}$.

$w^{\prime}=$ fluctuation of the z-component of the velocity, $\mathrm{m} / \mathrm{s}$.

$w_{1}=$ integration constant for grid turbulence (constant viscosity), $m^{-6}$.

$w_{2}=$ integration constant for grid turbulence (general viscosity), $\mathrm{m}^{6} / \mathrm{s}^{6}$.

$w_{3}=$ integration constant, $\mathrm{m}^{6} / \mathrm{s}^{6}$.

$w_{4}=$ integration constant, dimensionless.

$w_{5}=$ integration constant, dimensionless .

$w^{*}=$ dimensionless constant .

$x=$ space dimension, $m$.

$z=$ space dimension, $m$.

$z^{*}=$ nondimensional space dimension. 


\section{Greek Symbols}

$\alpha=$ modified turbulence Reynolds number.

$\varepsilon=$ turbulent energy dissipation rate, $\mathrm{m}^{2} / \mathrm{s}^{3}$.

$\varepsilon^{*}=$ nondimensional turbulent energy dissipation rate.

$\kappa=$ parameter of the elliptic integral, dimensionless

$v_{t}=$ turbulent kinematic viscosity, $\mathrm{m}^{2} / \mathrm{s}$.

$\nu=$ kinematic viscosity of the fluid, $\mathrm{m}^{2} / \mathrm{s}$.

$\sigma_{\varepsilon}=k-\varepsilon$ model constant for $\varepsilon$.

$\sigma_{k}=k-\varepsilon$ model constant for $k$.

$\varphi=$ parameter of the elliptic integral, dimensionless

\section{Subscripts}

0 relative to the origin position.

$1,2,3,4,5$ relative to integration constants or positions within the fluid.

$2 \varepsilon$ relative to the $\varepsilon$ equation.

500 relative to 500 images.

$f$ relative to longitudinal correlation.

$g$ relative to transversal correlation.

$k$ relative to kinetic energy.

$m$ relative to Matsunaga et al. transformation.

$N$ relative to the number of images.

$t$ relative to turbulence.

$\varepsilon$ relative do energy dissipation rate.

$\mu$ relative to viscosity.

\section{Theoretical Approximation Considering Constant $\boldsymbol{v}_{T}$}

Schulz and Chaudhry $(1998,1999)$ and Schulz (2001) presented analytical solutions for two-grid turbulence, assuming no mean flow and that the statistical quantities vary only along the $z$ axis (normal to the planes of the grids). For this field, considering first the hypothesis of a constant turbulent viscosity, $v_{t}$, the governing equations from the $k-\varepsilon$ model are given only by

$$
\frac{v_{t}}{\sigma_{k}} \frac{\partial}{\partial z}\left(\frac{\partial k}{\partial z}\right)=\varepsilon \quad \text { and } \quad v_{t}=C_{\mu} \frac{k^{2}}{\varepsilon}
$$

in which $k$ is the turbulent energy, $\varepsilon$ is the dissipation rate, and $\sigma_{k}$ and $C_{\mu}$ are model constants. Eliminating $\varepsilon$ and integrating the resulting equation, Eq. (2) and (3) are obtained:

$$
\begin{gathered}
\left(\frac{\partial^{2} k}{\partial z^{2}}\right)=C_{\mu} \frac{\sigma_{k}}{v_{t}^{2}} k^{2} \\
\frac{d}{d z}\left(\frac{C_{\mu} \sigma_{k}}{v_{t}^{2}} k\right)= \pm \sqrt{w_{l}+\frac{2}{3}\left(\frac{C_{\mu} \sigma_{k}}{v_{t}^{2}} k\right)^{3}}
\end{gathered}
$$

where $w_{l}$ is an integration constant. Considering that the turbulent kinetic energy assumes a minimum value $k_{0}$ at $z=0$ (the central position between the two grids), the boundary conditions are given by $k=k_{0}$ and $d k / d z=0$ at $z=0$. To simplify Eq (3), the following dimensionless quantities are defined ( $L$ is the half distance between grids and $R e$ is a convenient Reynolds number):

$$
\begin{gathered}
k^{*}=\frac{k}{k_{0}}, \quad s=\frac{z}{L}, \quad \alpha=\frac{L \sqrt{k_{0}}}{v_{t}} \sqrt{\frac{2 C_{\mu} \sigma_{k}}{3}} \text { or } \\
\alpha=\operatorname{Re} \sqrt{\frac{2 C_{\mu} \sigma_{k}}{3}} \quad \text { with } \operatorname{Re}=\frac{L \sqrt{k_{0}}}{v_{t}}
\end{gathered}
$$

These definitions and boundary conditions simplify Eq. (3) to

$$
\frac{d k *}{d s}= \pm \alpha \sqrt{k *^{3}-1}
$$

which integrated produces $\int_{1}^{k^{*}} \frac{d t}{\sqrt{k^{*}-1}}= \pm \alpha s$. The right term (with the integral symbol) is recognized as an elliptic integral of the first kind. Therefore, this equation can be represented as

$$
m F(\varphi, \kappa)= \pm \alpha s
$$

where $m, \varphi$ and $\kappa$ are the parameters of the elliptic integral $F(\varphi, \kappa)$. The values of $m, \varphi$ and $\kappa$ are required to visualize the evolution of the nondimensional turbulent kinetic energy $\left(k^{*}\right)$, with the nondimensional distance $(s)$. For the present case, the values are:

$$
m=\frac{1}{\sqrt[4]{3}} \cong 0.7598357, \kappa=\frac{\sqrt{2-\sqrt{3}}}{2} \cong 0.2588190, \cos \varphi=\frac{\sqrt{3}+1-k^{*}}{\sqrt{3}-1+k^{*}}
$$

Figure 1 shows the graph of Eq. (6) for $k^{*}$ as function of $\alpha$ s.

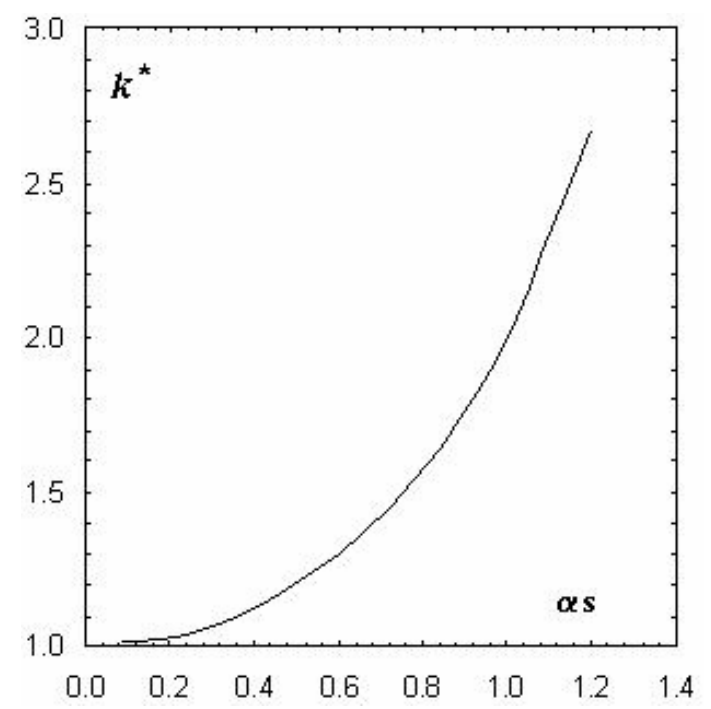

Figure 1. Curve obtained for $\mathrm{k}^{*}$ versus $\alpha \mathrm{s}$, from Eq. (6).

\section{Theoretical Approximation Considering General $v_{T}$}

Diffusion and dissipation of turbulence with a general turbulent viscosity may also be described by the $k$ - $\varepsilon$ model. In this case both transport equations, for $k$ and $\varepsilon$, must be used. Schulz and Chaudhry (1998, 1999) and Schulz (2001) proposed solutions for $k$ and $\varepsilon$ considering general turbulent viscosity, with the solutions presented as power series of the distance to the origin. In the present study an alternative solution is furnished, based on a linear differential equation relating $k$ and $\varepsilon$.

The original set of equations to be solved is given by Eq. (7) and (8) (two coupled nonlinear equations).

$$
\frac{\partial}{\partial z}\left(\frac{C_{\mu}}{\sigma_{k}} \frac{k^{2}}{\varepsilon} \frac{\partial k}{\partial z}\right)=\varepsilon
$$




$$
\frac{\partial}{\partial z}\left(\frac{C_{\mu}}{\sigma_{\varepsilon}} \frac{k^{2}}{\varepsilon} \frac{\partial \varepsilon}{\partial z}\right)=C_{2 \varepsilon} \frac{\varepsilon^{2}}{k}
$$

The first integration of Eq. (7) furnishes now (Schulz and Chaudhry, 1998):

$$
\varepsilon=\frac{d}{d z} \sqrt{\frac{2}{3} \frac{C_{\mu}}{\sigma_{k}}\left(k^{3}+w_{2}\right)}
$$

$w_{2}$ is an integration constant. Although Eq. (9) is still nonlinear, $\varepsilon$ and $k$ appear isolated (only one variable at each side of the equation), and suggest that

$$
\varepsilon=\varepsilon(k) \quad \text { and } \quad k=k(z)
$$

Matsunaga et al. (1999) proposed transformation (11), which simplify the representation of the original equations:

$$
\frac{d F_{m}}{d z}=\frac{\sigma_{k}}{v_{t}}
$$

Using Eq. (11) into Eq. (7) and (8) leads to:

$$
\begin{aligned}
& \frac{d^{2} \bar{k}}{d F_{m}{ }^{2}}=\bar{k}^{2} \\
& \frac{d^{2} \varepsilon}{d F_{m}{ }^{2}}=j \varepsilon \bar{k}
\end{aligned}
$$

where the following definitions apply:

$$
\bar{k}=\frac{C_{\mu}}{\sigma_{k}} k \quad \text { and } \quad j=\frac{C_{2 \varepsilon} \sigma_{\varepsilon}}{\sigma_{k}}
$$

The first integration of Eq. (12), after the suggestion of Schulz and Chaudhry (1998, 1999), furnishes:

$$
\frac{d \bar{k}}{d F_{m}}= \pm \sqrt{w_{3}+\frac{2}{3} \bar{k}^{3}}
$$

$w_{3}$ is an integration constant. Equations (9) and (10) are still valid, so that we have:

$$
\varepsilon=\varepsilon(\bar{k}) \quad \text { and } \quad \bar{k}=\bar{k}\left(F_{m}\right)
$$

From Eq. (17) it follows immediately that:

$$
\frac{d^{2} \varepsilon}{d F_{m}{ }^{2}}=\frac{d^{2} \varepsilon}{d \bar{k}^{2}}\left(\frac{d \bar{k}}{d F_{m}}\right)^{2}+\frac{d \varepsilon}{d \bar{k}} \frac{d^{2} \bar{k}}{d F_{m}{ }^{2}}
$$

Using Eq. (12), (13) and (15) into Eq. (18) leads to:

$$
\left(w_{3}+\frac{2}{3} \bar{k}^{3}\right) \frac{d^{2} \varepsilon}{d \bar{k}^{2}}+\bar{k}^{2} \frac{d \varepsilon}{d \bar{k}}-j \bar{k} \varepsilon=0
$$

This result is important, because the original problem, composed by two coupled nonlinear equations for $\varepsilon$ and $\kappa$ is transformed into only one linear equation for $\varepsilon=\varepsilon(\bar{k})$. To generalize the equation and its results, a nondimensional form (Eq. 20) is presented, considering the values of the turbulent kinetic energy $\left(k_{0}\right)$ and its dissipation rate $\left(\varepsilon_{0}\right)$ at the origin.

$$
\begin{gathered}
\left(w^{*}+\frac{2}{3} k^{*}\right) \frac{d^{2} \varepsilon^{*}}{d k^{2}}+k^{2} \frac{d \varepsilon^{*}}{d \bar{k} *}-j k * \varepsilon^{*}=0 \\
\varepsilon^{*}=\frac{\varepsilon}{\varepsilon_{0}} \quad \text { and } \quad k^{*}=\frac{k}{k_{0}}
\end{gathered}
$$

$w^{*}$ is a nondimensional constant. A solution of this linear equation is presented using Hypergeometric functions (Hyp2Fl in Eq. 21), useful to represent turbulence generated in the region between two oscillating grids.

$$
\varepsilon^{*}(k *)=\frac{\left(\begin{array}{l}
\text { Hyp } 2 F 1\left[-0.567,0.734,0.667,-\frac{0.667 k^{*}}{w^{*}}\right] \\
k * w_{5} H y p 2 F 1\left[-0.567,0.734,0.667,-\frac{0.667}{w^{*}}\right] \operatorname{Hyp} 2 F 1\left[-0.234,1.067,1.333,-\frac{0.667 k^{* 3}}{w^{*}}\right]
\end{array}\right)}{\text { Hyp } 2 F 1\left[-0.567,0.734,0.667,-\frac{0.667}{w^{*}}\right]}
$$

$w_{4}$ and $w_{5}$ are integration constants

\section{Experimental Methods}

Figure 2 shows the turbulence-generating system used in this study, a tank with walls of acrylic plates, with a $0.50 \mathrm{~m} \times 0.50 \mathrm{~m}$ square cross-section and $1.15 \mathrm{~m}$ height. The transparent walls allow laser beams to be projected into the water and the resulting images to be captured with a CCD camera. These images were used to evaluate instantaneous velocity fields. Two identical horizontally oriented grids, $31.6 \mathrm{~cm}$ apart from each other, were coupled to a vertically oscillating driving device and oscillated in phase. The lower grid was positioned $55.0 \mathrm{~cm}$ above the bottom of the tank. The grids were built using $1.0 \mathrm{~cm}$ square bars with a $5.1 \mathrm{~cm}$ mesh size $(M)$, obtaining a solidity of $32 \%$. A $0.2-0.3 \mathrm{~cm}$ gap between the sidewalls and the grids permitted the easy manipulation of the grids in the tank. The stroke $S$ was varied from 2.0 to $5.0 \mathrm{~cm}$ and the frequency $f$ from 1.0 to $4.0 \mathrm{~Hz}$. Table 1 presents experimental parameters, where $\alpha$ is described in detail in the Section "Experimental Results", and is used to obtain Fig. 10. Oscillating grid turbulence is sensitive to initial conditions, so that data acquisition could only begin $30 \mathrm{~min}$ after the onset of oscillation.

As mentioned, the velocity fields were measured by the DPIV technique (Digital Particle Image Velocimetry). Details are found in Janzen (2003). Images of approximately $15.0 \mathrm{~cm} x 15.0 \mathrm{~cm}$ were taken at the middle distance between the grids. The "interrogation area" chosen to evaluate the vectors was about $1.0 \mathrm{~cm} x 1.0 \mathrm{~cm}(32 x$ 32 pixels) with a $50 \%$ overlap. About 500 image pairs were taken 
for each experiment. An example of the variation of the turbulence intensities $(u$ and $w$ ), at different distances $z$, with the number of images, is shown in Fig. 3. It shows that the results are statistically consistent for a number of images higher than approximately 300 . The images were captured in sets of 16 photos, with a velocity of 30 photos per second, during a total period of about 5.0 minutes. For each image, $29 \times 29$ velocity vectors were calculated. Additionally, computations were performed to evaluate temporally averaged characteristics of turbulence for all the points in the imaged area. To compare these experimental results with the one-dimensional analytical predictions, the data of the turbulence intensities were averaged along planes parallel to the grids, i.e., mean values were obtained for each $z$. For the present study, the turbulent energy $k$ was evaluated as $k=\left(2 \overline{u^{\prime 2}}+\overline{w^{\prime 2}}\right) / 2$, where $u^{\prime}$ and $w^{\prime}$ are, respectively, the horizontal and vertical instantaneous velocity fluctuations.

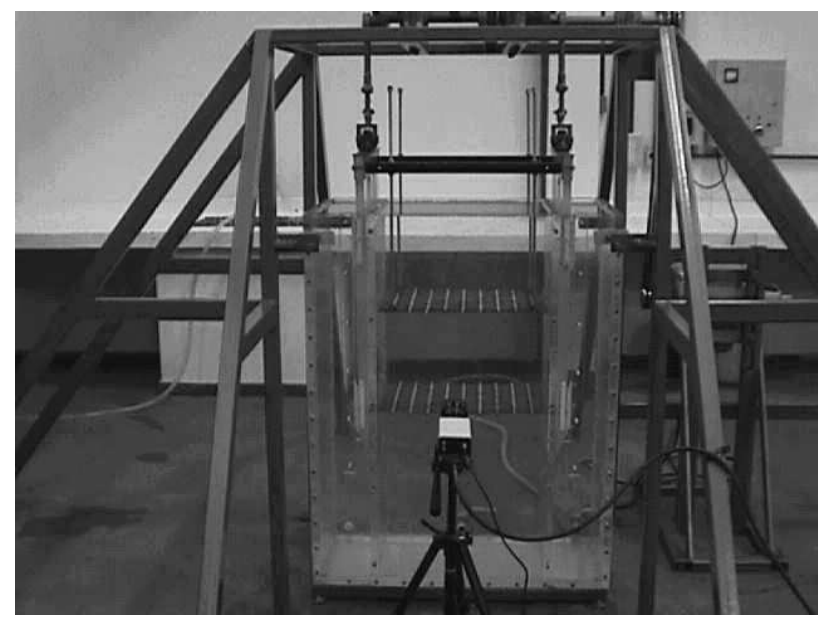

Figure 2. Experimental apparatus. The distance between the grids is $31.6 \mathrm{~cm}$.

Table 1. Experimental conditions used to conduct the experiments.

\begin{tabular}{|c|c|c|c|c|c|c|}
\hline Run no. & $f(\mathbf{H z})$ & $S(\mathrm{~cm})$ & $\mathrm{fS}^{2} / v$ & $\alpha$ & \multicolumn{2}{|c|}{$L(\mathrm{~cm})$} \\
\hline \multirow{2}{*}{1} & \multirow{2}{*}{2.0} & \multirow{2}{*}{2.0} & \multirow{2}{*}{800} & 1.99 & 14.52 & 1 \\
\hline & & & & 1.99 & 6.34 & 2 \\
\hline \multirow{2}{*}{2} & \multirow{2}{*}{3.0} & \multirow{2}{*}{2.0} & \multirow{2}{*}{1200} & 1.61 & 15.57 & 1 \\
\hline & & & & 1.63 & 5.28 & 2 \\
\hline \multirow{2}{*}{3} & \multirow{2}{*}{4.0} & \multirow{2}{*}{2.0} & \multirow{2}{*}{1600} & 1.52 & 13.46 & 1 \\
\hline & & & & 1.68 & 7.39 & 2 \\
\hline \multirow{2}{*}{4} & \multirow{2}{*}{1.0} & \multirow{2}{*}{4.0} & \multirow{2}{*}{1600} & 1.60 & 13.05 & 1 \\
\hline & & & & 2.18 & 6.15 & 2 \\
\hline \multirow{2}{*}{5} & \multirow{2}{*}{2.0} & \multirow{2}{*}{4.0} & \multirow{2}{*}{6400} & 1.86 & 12.54 & 1 \\
\hline & & & & 2.37 & 6.15 & 2 \\
\hline \multirow{2}{*}{6} & \multirow{2}{*}{3.0} & \multirow{2}{*}{4.0} & \multirow{2}{*}{4800} & 2.13 & 13.05 & 1 \\
\hline & & & & 1.77 & 5.13 & 2 \\
\hline \multirow{2}{*}{7} & \multirow{2}{*}{1.0} & \multirow{2}{*}{5.0} & \multirow{2}{*}{2500} & 1.98 & 14.78 & 1 \\
\hline & & & & 1.77 & 4.20 & 2 \\
\hline \multirow{2}{*}{8} & \multirow{2}{*}{2.0} & \multirow{2}{*}{5.0} & \multirow{2}{*}{5000} & 1.84 & 12.68 & 1 \\
\hline & & & & 1.66 & 5.77 & 2 \\
\hline \multirow{2}{*}{9} & \multirow{2}{*}{3.0} & \multirow{2}{*}{5.0} & 7500 & 1.63 & 14.78 & 1 \\
\hline & & & & 1.75 & 3.67 & 2 \\
\hline
\end{tabular}




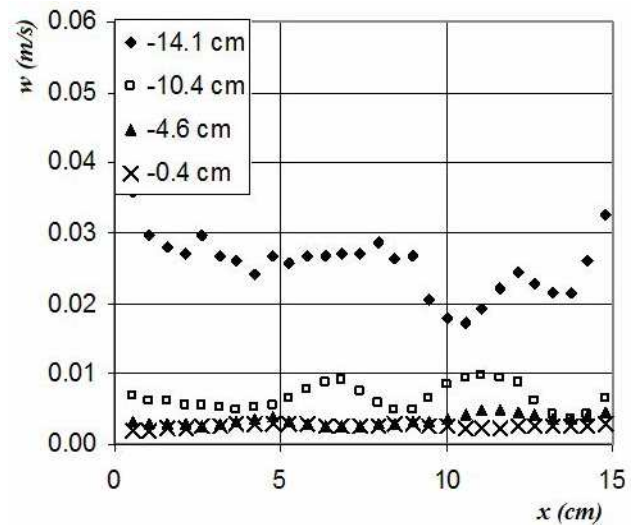

(b)

Figure 4. (Continued)

Horizontal distributions of Reynolds stresses are shown in Fig. 5. Variations of $\overline{u^{\prime} w^{\prime}}$ near the grids can be noted, i.e., shear flow exists at small distances from the grids. Turbulence can be considered shear-free only at greater distances from the grid (Reynolds stresses approach zero). The data suggest that the turbulence may be isotropic in the sense that the Reynolds stresses are small (close to zero).

To obtain the integral length scale, the correlation functions were first calculated. The longitudinal $f(r)$ and transversal $g(r)$ correlation functions are defined as:

$$
f(r)=\frac{\overline{u^{\prime}(x) u^{\prime}(x+r)}}{u(x) u(x+r)} \quad \text { and } \quad g(r)=\frac{\overline{w^{\prime}(x) w^{\prime}(x+r)}}{w(x) w(x+r)}
$$

Figure 6 presents the longitudinal and transversal functions computed for $f=3.0 \mathrm{~Hz}$ and $S=5.0 \mathrm{~cm}$. It can be seen that the values increase with increasing distance from the grid, until $r$ about 10. The integral length scale is computed using the longitudinal and transversal correlation functions, as follows:

$$
L_{f}=\int_{0}^{\infty} f(r) d r \quad \text { and } \quad L_{g}=\int_{0}^{\infty} g(r) d r
$$

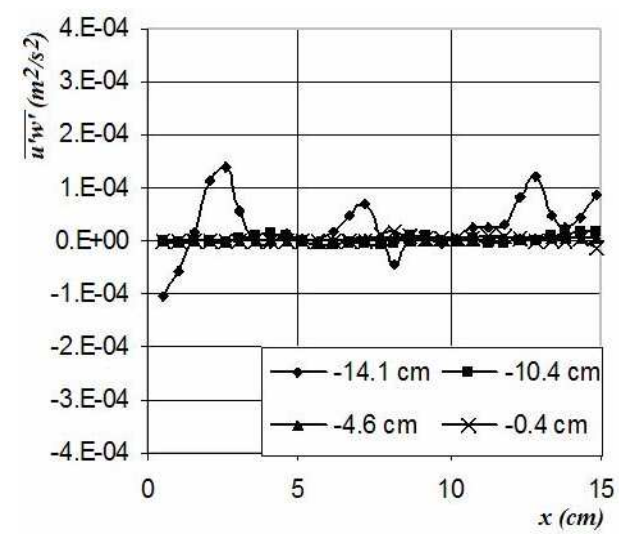

(a)

Figure 5. Reynolds stresses along $x$ axis for different distances $z$ : (a) $S=$ $4.0 \mathrm{~cm} ; \mathrm{f}=1.0 \mathrm{~Hz}$ (b) $\mathrm{S}=4.0 \mathrm{~cm} ; \mathrm{f}=2.0 \mathrm{~Hz}$.

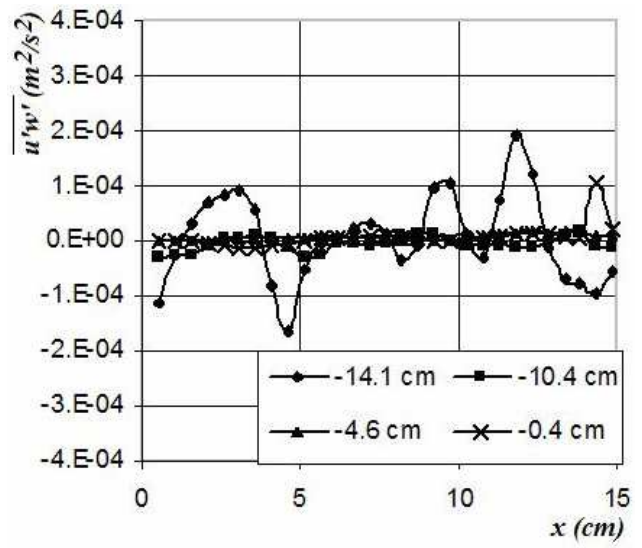

(b)

Figure 5. (Continued).

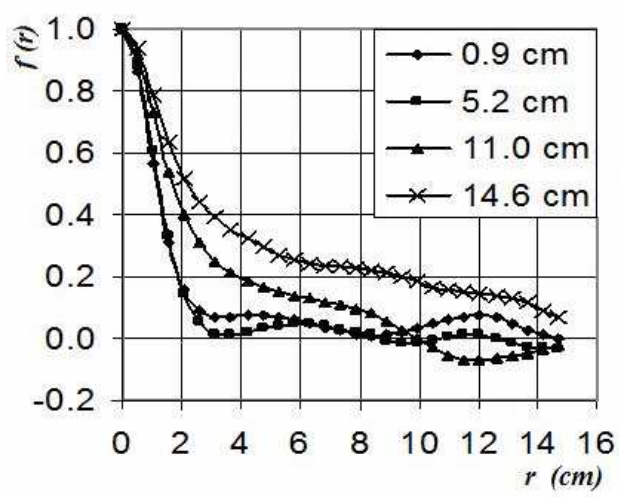

(a)

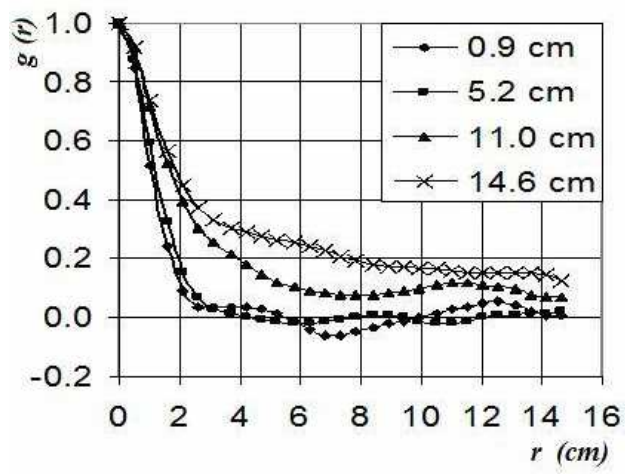

(b)

Figure 6. (a) Longitudinal and (b) transverse correlation functions at different distances $z(S=5.0 \mathrm{~cm} ; f=3.0 \mathrm{~Hz})$.

A total integral length scale was calculated, because the grid turbulence is not completely isotropic, using the longitudinal and transversal scales, as $L_{t}=\sqrt{2 L_{f}^{2}+L_{g}^{2}}$. The total integral length scale $L_{t}$ is plotted against the distance $z$ in Fig. 7 for $S=5.0 \mathrm{~cm}$ and $f=3.0 \mathrm{~Hz}$. The data suggest a combination of two linear trends, one for the upper side of the region between the grids and the other for the lower side. The same behavior is obtained for the other runs. 


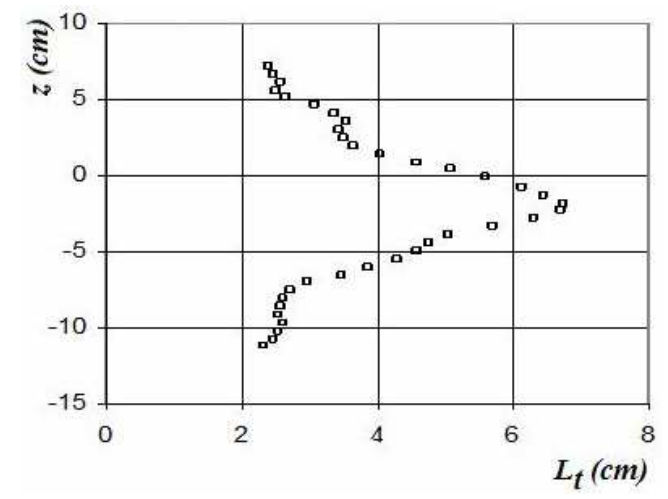

Figure 7. Relation between $z$ and Lt $(S=5.0 \mathrm{~cm} ; f=3.0 \mathrm{~Hz})$.

Experimental data of $v_{t}$ are plotted against $z$ in Fig. 8. It can be seen that the turbulent viscosity is approximately constant in the central region between the grids, but increases strongly in the vicinity of the grids.

These observations indicate that the assumption of constant turbulent viscosity, used in the first analytical solution presented here, is an acceptable approximation for the central region. For the entire space between the grids it is better to use the general turbulent viscosity solution. Further, Fig. 9 presents the turbulent kinetic energy $k$ against the distance $z$ for $S=4 \mathrm{~cm}$ and $f=3 \mathrm{~Hz}$. Interesting "S forms" are observed for the behavior of $k$ along $z$ near the grids, which may be related to production and advection of kinetic energy in this region. As the theoretical models considered until now encompasses (embraces) only the diffusive-dissipative phenomena (without production and advection), the comparisons between experimental and theoretical results must be considered for the central region, where production does not play an important role. It can also be seen, in Fig. 9, that the minimum of the turbulent kinetic energy, ideally at $z=0$, is dislocated in about $2.3 \mathrm{~cm}$. Such dislocation was also observed by Srdic et al. (1996) and Ott and Mann (2000). To compare the present results with the analytical ones, the $z$ axis was submitted to a translation in $2.3 \mathrm{~cm}$, so that the origin of $z$ coincides with the minimum of the turbulent kinetic energy. This translation of the axis appoints the necessity to calculate two $\alpha$ values, since the distance $L$ from the point of minimum to both grids is not the same anymore. These distances are indicated as $L_{2}$ (upper region) and $L_{1}$ (lower region) in Table 1 . The least squares method was used to fit the $\alpha$ value for each situation. The values of $\alpha$ and the different distances $L$ are given in Table 1 . Figure 10 presents the good agreement for $k^{*}$ and $s$ between experimental data and the analytical solution (Eq. 6) for different experimental conditions. The profile of $k^{*}$, obtained theoretically, hold very well to that of the experimental data, for $\alpha s$ values less than about 1.4, i.e., the region where the turbulent viscosity can be considered constant. The experimental data from Shy et al. (1997) and Ott and Mann (2000) are also presented.

As theoretical results using a general viscosity are also furnished, the comparison between predictions of $k$ and $\varepsilon$ and the experimental data is also possible. Figures 11 and 12 show the agreement between the proposed solution of Eq. (21) and experimental data obtained by Janzen (2003), for the experimental conditions already described in Table 1 . The experimental data for the situations of minimum and maximum stroke were compared with the theoretical predictions in nondimensional form.

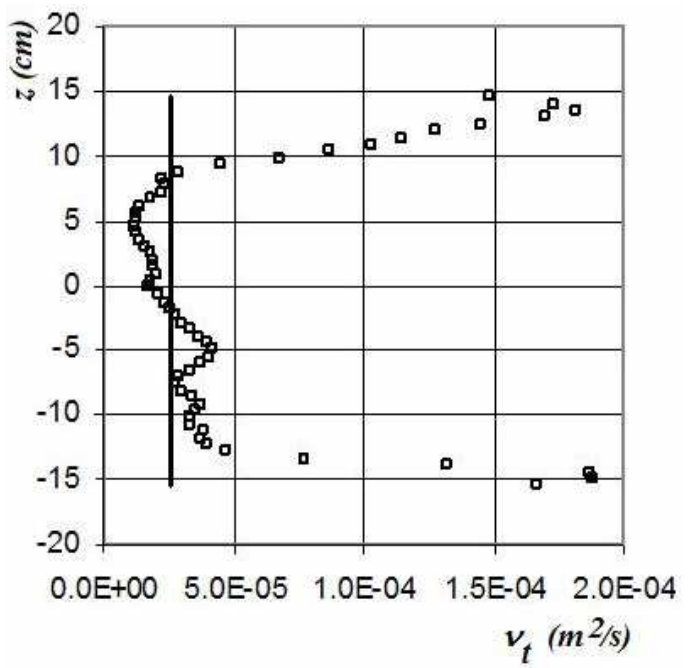

(a)

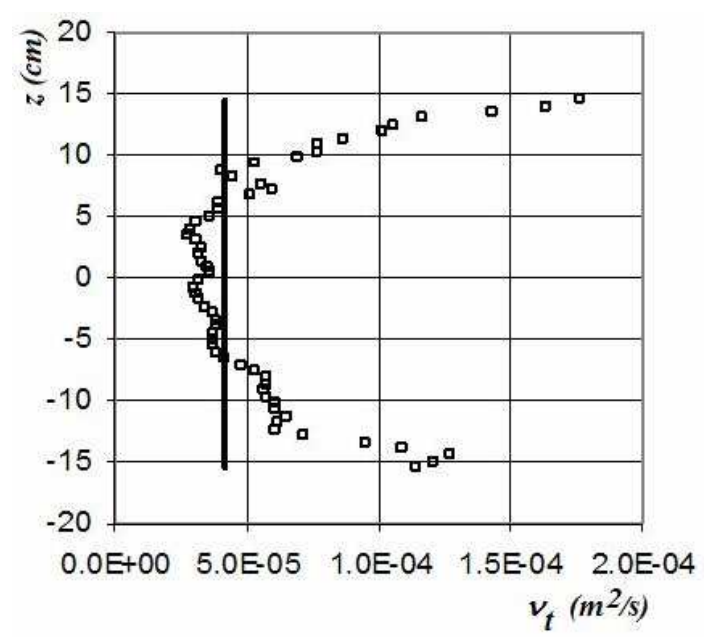

(b)

Figure 8. Relation between $\square t$ and $z$ for (a) $S=5.0 \mathrm{~cm} ; f=1.0 \mathrm{~Hz}$ and (b) $\mathrm{S}$ $=5.0 \mathrm{~cm} ; f=2.0 \mathrm{~Hz}$. Solid lines represent mean "constant" viscosities.

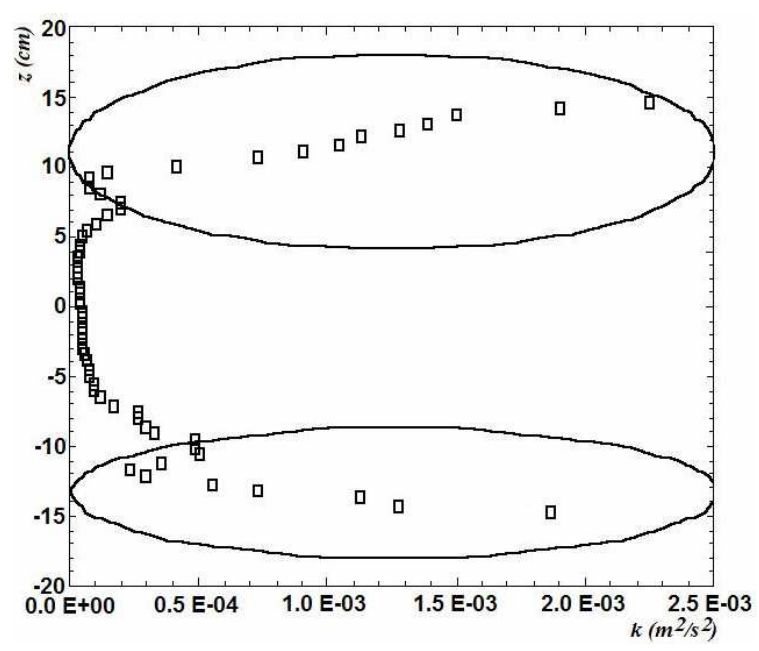

Figure 9. Turbulent kinetic energy $k$ versus $z$ for $S=4.0 \mathrm{~cm}$ and $f=3.0 \mathrm{~Hz}$. "S forms" are observed for $k$ along $z$ that the minimum of the turbulent kinetic energy, ideally at $z=0$, is dislocated. 


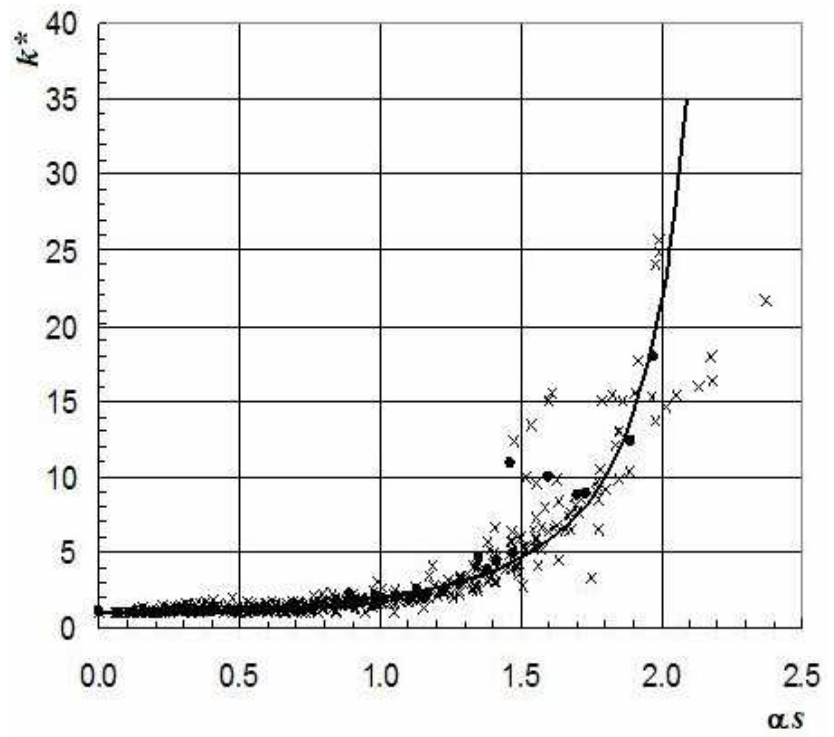

Figure 10. Experimental data and analytical solution ( $\left.\alpha s, k^{*}\right)$. Solid curve obtained from Eq. (6). (x) Present authors. ( $\Delta$ ) Ott and Mann (2000)(these data are very close to the origin and are not visible in the figure). (๑) Shy et al. (1997).

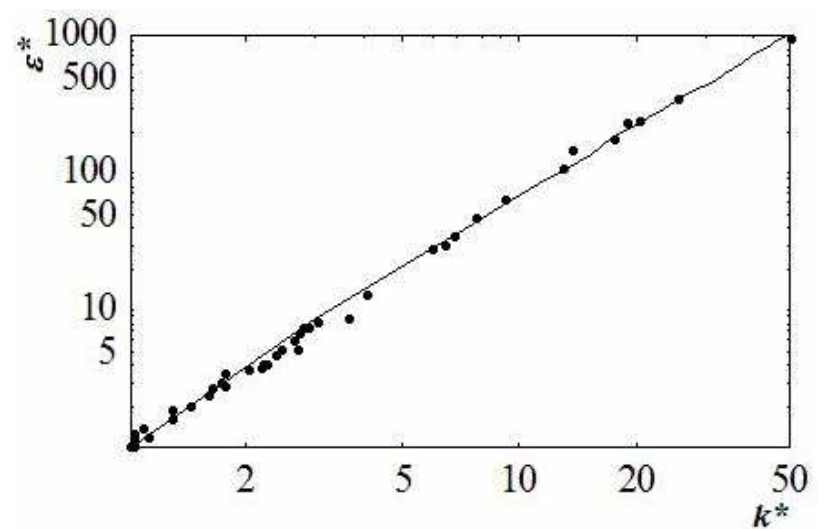

Figure 11. $\varepsilon^{\star}$ versus $k^{\star}(S=2.0 \mathrm{~cm}, f=2.0 \mathrm{~Hz}$ of Table 1$)$. The line is the prediction of Eq. (21). Dots are measured values.

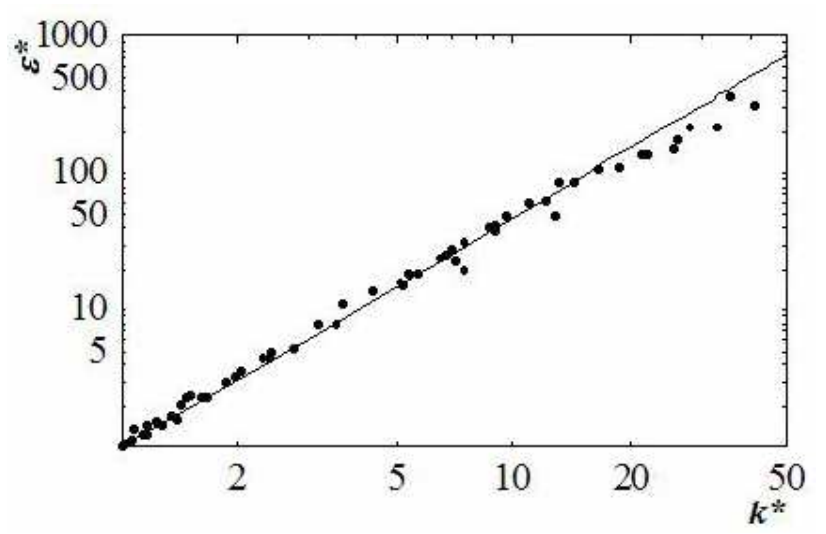

Figure 12. $\varepsilon^{*}$ versus $k^{*}(S=5.0 \mathrm{~cm}, f=2.0 \mathrm{~Hz}$ of Table 1$)$. The line is the prediction of Eq. (21). Dots are measured values.

The conditions used for the present analysis are specified in Table 2. Such analysis was also performed, in dimensional form, by
Souza (2004). The constants of the $k$ - $\varepsilon$ model used here are $C,=1.44$, $C_{2}=1.92, C_{\mu}=0,09, \sigma_{\varepsilon}=1.3$ and $\sigma_{k}=1.0$. The boundary condition adopted is: for $k^{*}=1, \varepsilon^{*}=1$. The remaining integration constants were evaluated through adjustment with the experimental data.

Table 2. Maxima and minima conditions for the stroke in the present study, used to evaluate the predictions of Eq. 21.

\begin{tabular}{|c|c|c|c|}
\hline $\begin{array}{c}\text { Frequency } \boldsymbol{f} \\
(\mathrm{Hz})\end{array}$ & $\begin{array}{c}\text { Stroke } \boldsymbol{S} \\
(\mathrm{cm})\end{array}$ & $\boldsymbol{w}_{4}=\boldsymbol{w}_{5}$ & $\boldsymbol{w}^{*}$ \\
\hline $\mathbf{2 . 0}$ & 2.0 & 4.5 & 1.0 \\
\hline $\mathbf{3 . 0}$ & 2.0 & 6.0 & 1.0 \\
\hline $\mathbf{2 . 0}$ & 5.0 & 2.0 & 1.0 \\
\hline $\mathbf{3 . 0}$ & 5.0 & 2.5 & 1.0 \\
\hline
\end{tabular}

Hypergeometric functions as presented by Eq. (21) are tabulated in the literature and may also be evaluated through adequate softwares. The obtained solution permits, of course, to calculate the spatial evolution of the relevant parameters (although numerically). As an example, the equations presented in this study permit to obtain the evolution of $k^{*}$ with the nondimensional distance $z^{*}$, in the form of Eq. (24):

$$
\frac{d z^{*}}{d k^{*}}=\left(\frac{C_{\mu}}{\sigma_{k}}\right)^{0.5} \frac{k^{* 2}}{\varepsilon^{*}\left(k^{*}\right) \sqrt{w^{*}+\frac{2}{3} k^{* 3}}}, \text { where } z^{*}=\frac{z \varepsilon_{0}}{k_{0}^{3 / 2}}
$$

$\varepsilon^{*}\left(k^{*}\right)$ is given by Eq. (21). A graph of $k$ against $z^{*}$, for the same condition of Fig. 11, is given by Fig. 13. Equation (24) was solved using a fourth order Runge-Kutta scheme, with boundary condition $k^{*}=1$ for $z^{*}=0$. Experimental energy dissipation rates were obtained using $\varepsilon=C_{D} k^{3 / 2} / L_{t}$, where $C_{D}$ is an empirical constant and $L_{t}$ is the integral turbulence length scale. The value of $C_{D}$ used here was 0.5 . Figure 13 also contains the points located closer to the grids, marked with ellipses in Fig. 9a. It can be seen that the agreement between theory and experimental data is also good for the general turbulent viscosity case, as quantified here.

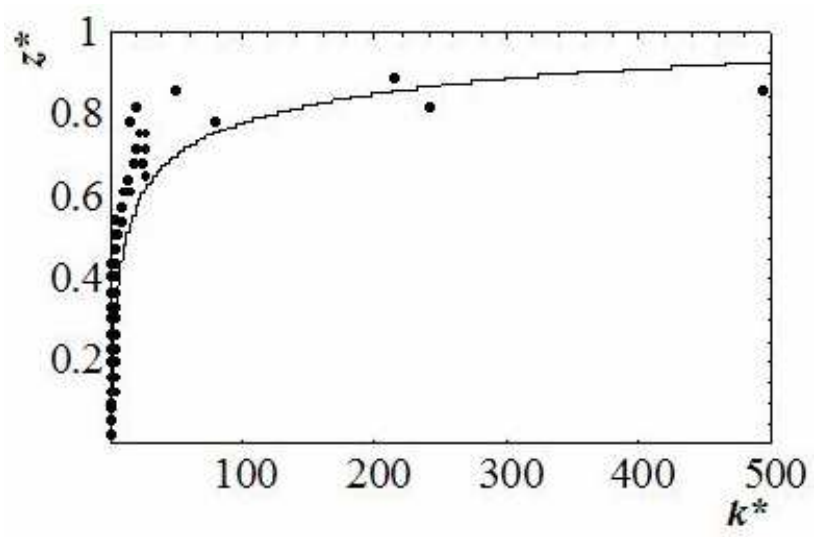

Figure 13. $k^{*}$ as function of the normalized distance $z^{*}(S=2.0 \mathrm{~cm}, f=2.0$ $\mathrm{Hz}, \mathrm{C}_{\mathrm{D}}=0.5$ ). The line is the prediction of Eq. (24). Dots are measured values.

\section{Conclusions}

The turbulence field generated by a pair of grids was studied experimentally and theoretically. Theoretical predictions obtained through application of the $k-\varepsilon$ model were compared with the experimental data. The solution for the behavior of the turbulent kinetic energy, considering constant turbulent viscosity, is supported 
by the experimental data for the central region between the grids. It is therefore possible to predict the behaviour of $k$ and $\varepsilon$ for turbulence generated by a pair of oscillating grids assuming constant $v_{t}$ around the central region of the flow. Furthermore, the present study reveals that the integral length scale has an approximately linear behavior with the distance to the origin. The measurements also suggest that the turbulence produced by a pair of oscillating grids can be considered nearly isotropic in the sense that the Reynolds stresses are small.

Furthermore, considering the equations for the turbulent field presented here using a general turbulent viscosity, it is important to stress that the original set of equations composed by two coupled nonlinear differential equations was transformed, through mathematical tools, into a single linear differential equation relating $k$ and $\varepsilon$. A solution of this governing equation was presented, and the theoretical prediction agrees well with experimental data. In this case, the behaviour of $k$ could be followed until greater distances to the origin (in comparison with the solution for constant turbulent viscosity). For both cases, constant and general turbulent viscosity, the equations consider diffusion and dissipation of $k$ and $\varepsilon$, which is a situation more adequate for the central region between the grids.

\section{Acknowledgements}

The authors are indebted to FAPESP (Fundação de Amparo à Pesquisa do Estado de São Paulo), CNPq (Conselho Nacional de Pesquisa e Desenvolvimento Tecnológico) and CAPES (Coordenação de Aperfeiçoamento de Pessoal do Ensino Superior), Brazilian research support foundations.

\section{References}

Brodkey, R.S. ,1967, "The Phenomena do Fluid Motions", AddisonWesley Publishing Company, Library of Congress Catalog Card No. 6714206, U.S.A.

Brunk, B., Shirk, M.W., Jensen, A., Jirka, G. \& Lion, L.W., 1996, "Modeling natural hydrodynamic systems with a differential-turbulence column”, Journal of Hydraulic Engineering, Vol.122, pp 373-380.

De Silva, I.P.D. \& Fernando, H.J.S., 1994, "Oscillating grids as a source of nearly isotropic turbulence”, Phys. Fluids, Vol.6, pp 2455-2464.

Cheng, N.S. \& Law, W.K., 2001, "Measurements of turbulence generated by oscillating grid", Journal of Hydraulic Engineering, Vol. 127, No.3, p.201-208.
Hopfinger, E.J. \& Toly, J.A., 1976, "Spatially decaying turbulence and its relation to mixing across density interfaces", J. Fluid Mechanics, Vol. 78, pp 155-175.

Janzen, J.G.,2003," Details of turbulence properties in water agitated through a pair of oscillating grids", Master degree thesis, School of Engineering at São Carlos, University of São Paulo, Brazil (in Portuguese).

Janzen, J.G. \& Schulz, H.E.,2003, "Using PIV to determine turbulence characteristics in tanks with two oscillating grids", Proceedings of the 15th Brazilian Symposium on Water Resources, ABRH, Published in CD-ROM, Curitiba PR, Brazil (in Portuguese), 23 to 27 November.

Matsunaga, N.; Sugihara, Y.; Komatsu, T. \& Masuda, A., 1999, "Quantitative properties of oscillating-grid turbulence in a homogeneous fluid”, Fluid Dynamics Research, Vol. 25, pP.147-165.

Schulz, H.E. \& Chaudhry, F.H., 1998, "A Theoretical Solution for Turbulence Generated by Oscillating Grids", Proceedings of the First Spring School of Transition and Turbulence, Brazilian Association of Mechanical Sciences, COPPE, Rio de Janeiro, Brazil, 21 to 25/09/1998, pp. 181-194.

Schulz, H.E., \& Chaudhry, F.H., 1999, "Theoretical Solutions for Turbulence Generated by Two Oscillating Grids. 15th Brazilian Congress of Mechanical Engineering. ISBN-85-85769-03-3, Published in CD-ROM Águas de Lindóia, SP, Brazil.

Schulz, H.E., 2001, "Alternatives in Turbulence"(in Portuguese). Ed. EESC-EDUSP, ISBN 85-85205-37-7, São Carlos, S.P., Brazil.

Shy, S.S, Jang, R.H. \& Tang, C.Y., 1996, "Simulation of turbulent burning velocities using aqueous autocatalytic reactions in a nearhomogeneous turbulence", Combustion and Flame, Vol. 105, pp 54-67.

Shy, S.S, Tang, C.Y. \& Fann, S.Y., 1997, “A nearly isotropic turbulence generated by a pair of vibrating grids", Experimental Thermal and Fluid Science, Vol. 14, pp 251-262.

Souza, K.C.O., 2004, "Evaluation of theoretical solutions for turbulent flows", Master degree thesis, School of Engineering at São Carlos, University of São Paulo, Brazil (in Portuguese).

Souza, L.B.S., 2002, "Study of the turbulent structure in flows generated by oscillating grids", Master degree thesis, School of Engineering at São Carlos, University of São Paulo, Brazil (in Portuguese).

Srdic, A., Fernando, H.J.S. \& Montenegro, L., 1996, "Generation of nearly isotropic turbulence using two oscillating grids", Experimental in Fluids, Vol. 20, pp 395-397.

Ott, S. \& Mann, J., 2000, “An experimental investigation of the relative diffusion of particle pairs in three-dimensional turbulent flow", Journal of Fluid Mechanics, Vol.422, pp. 207-223.

Thompson, S.M \& Turner, J.S, 1975, "Mixing across an interface due to turbulence generated by an oscillating grid", J. Fluid Mechanics, Vol. 67, pp 349-368.

Townsend, A. A., 1976, "Homogeneous turbulent flows", In: The structure of turbulent shear flow. Cambridge, Cambridge University Press. pp. 45-103.

Villermaux, E., Sixou, B. \& Gagne, Y., 1995, "Intense vortical structures in grid-generated turbulence", Phys. Fluids, Vol. 7, pp. 2008-2013. 